\title{
Does early surgical repair of hip fractures improve patient outcomes?
}

Orosz GM, Magaziner J, Hannan EL, Morrison RS, Koval K, Gilbert $M$, et al. Association of timing of surgery for hip fracture and patient outcomes. JAMA 2004;291:1738-43.
Background: Over 220000 hip fractures occur every year in the United States, representing an annual $\$ 9$ billion cost to the health care system. ${ }^{1}$ Although several studies have evaluated prognostic factors in hip fracture management, physicians continue to debate whether early versus delayed operative stabilization improves patient outcomes. Proponents of early stabilization believe that complications from bed rest (i.e., thromboembolism, pressure ulcers, urinary tract infections) can be reduced; however, others support a surgical delay to allow optimization of a patient's preoperative medical status.

Design: In this prospective multicentre cohort study, the investigators examined outcomes of patients aged 50 years or more who had early (in 24 hours or less after hospital arrival) surgical repair of a hip fracture compared with those who had later surgery. $\mathrm{Pa}-$ tients who had multiple injuries, pathological fractures, bilateral $\checkmark$ fractures or fractures of the femoे oral shaft or distal femur, and those who had previous surgery at the fracture site were excluded. $\approx$ Outcome measures were mean pain scores over the first 5 postoperative days, major complica๑ tions, length of hospital stay, death within 6 months and functional status at 6 months.

Results: Of the 1178 patients who had surgical repair of their hip fracture, 398 (33.8\%) had surgery within 24 hours, and 780 (66.2\%) had later surgery. Data on death and functional status at 6 months were available for $94 \%$ of the patients. The overall unadjusted mortality was $17.5 \%$ at 6 months. Early surgery was not associated with a decreased risk of death (hazard ratio [HR] 0.75 , $95 \%$ confidence interval [CI] 0.52 to 1.08 ) or of major postoperative complications (HR 0.55, $95 \%$ CI 0.24 to 1.1$)$. Other outcomes are shown in Table 1.

Commentary: The timing of surgery to repair hip fractures remains a controversial but important issue. Since no randomized trials exist, current evidence is based on observational data. This well-designed, large observational study sheds further light on the current debate. The study is strengthened by a comprehensive assessment of clinically meaningful outcomes and supplemental analyses to test the validity of the results. ${ }^{2}$ Observational datasets are limited when baseline differences between groups arise. In the current study, 10 baseline character-
Table 1: Adjusted outcomes* of patients undergoing early or late surgical repair of hip fracture

\begin{tabular}{lcccr}
\hline Variable & $\begin{array}{c}\text { Surgery } \\
\leq 24 \mathrm{~h}\end{array}$ & $\begin{array}{c}\text { Surgery } \\
>24 \mathrm{~h}\end{array}$ & Difference $(95 \% \mathrm{Cl})^{*}$ & $p$ value \\
\hline Pain score, ${ }^{*}$ mean & 2.63 & 2.87 & $-0.24(-0.44$ to -0.06$)$ & 0.01 \\
Length of stay, mean, d & 7.35 & 9.29 & $-1.94(-2.82$ to -1.06$)$ & $<0.01$ \\
FIM score at 6 mo & & & & \\
$\quad$ Locomotion (range 2-14) & 8.81 & 8.85 & $-0.04(-0.49$ to 0.39$)$ & 0.84 \\
$\quad$ Self care (range 6-42) & 30.3 & 31.4 & $-1.1(-2.18$ to -0.04$)$ & 0.04 \\
$\quad$ Transferring (range 3-21) & 13.6 & 13.9 & $-0.3(-0.92$ to 0.25$)$ & 0.26 \\
\hline
\end{tabular}

Note: FIM = Functional Independence Measure.

*Adjusted for age, sex, nursing home residence, needing a proxy for consent, delirium on admission, prefracture FIM locomotion score, fracture type, history of diabetes, chronic obstructive pulmonary disease, stroke syndrome, dementia, cardiac disease, hypertension, admission to hospital within 6 months, hospital site, day and time of admission, and abnormal clinical (physical examination and laboratory) findings.

†Pain scores range from 1 (none) to 5 (very severe pain). istics differed between the 2 patient groups, although the authors adjusted for them in their analysis. The analysis, however, cannot exclude a clinically important reduction in mortality with early surgery (up to $48 \%$ ). Although the $95 \%$ CI crosses equivalence, the current data support anywhere from a $1 \%$ to $50 \%$ reduction in mortality with $86 \%$ confidence. ${ }^{3}$ The definition of early surgery (in the first 24 hours after hospital arrival) also seems somewhat arbitrary. Despite the authors' assertion that 6 months of follow-up was sufficient, recovery following hip fractures may extend to 1 year. In a recent study involving 651 patients with hip fractures, early surgery (within 48 hours) significantly reduced the risk of death by 39\% (95\% CI $10 \%-58 \%$ ) at 1 year. $^{4}$

Practice implications: Physicians should aim for early operative fixation of hip fractures to reduce patients' pain and length of hospital stay. Larger studies will be needed to address whether early surgery reduces mortality and the incidence of major postoperative complications.

\section{Mohit Bhandari}

Department of Clinical

Epidemiology and Biostatistics

Division of Orthopaedic Surgery

McMaster University

Hamilton, Ont.

\section{References}

1. Ray NF, Chan JK, Thamer M, Melton LJ III. Medical expenditures for the treatment of osteoporotic fractures in the United States in 1995: report from the National Osteoporosis Foundation. 7 Bone Miner Res 1997;12:24-35.

2. Rubin DB. Estimating causal effects from large data sets using propensity scores. Ann Intern Med 1997;127:757-63.

3. Shakespeare T, Gebski VJ, Veness M, Simes J. Improving interpretation of clinical studies by use of confidence levels, clinical significance curves, and risk-benefit contours. Lancet 2001; 357:1349-53

4. Gdalevich M, Cohen D, Yosef D, Tauber C. Morbidity and mortality after hip fracture: the impact of operative delay. Arch Orthop Trauma Surg 2004;124:334-40. 